\title{
The Status Quo and Development of Study on the Prevention and Countermeasures against the Non-traditional Security Threats in Xinjiang
}

\author{
Liang Xiong ${ }^{1,2, a}$, Weiwei Wang ${ }^{1, b}$, Silan $\mathrm{Li}^{1, \mathrm{c}}$, Xin $\mathrm{Xu}^{1, \mathrm{~d}}$ \\ ${ }^{1}$ Military Economics Academy, Wuhan,430035, China \\ ${ }^{2}$ Social Science Academy of Xinjiang,Urumqi,830001, China

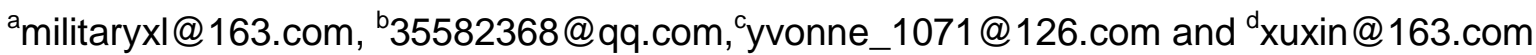

Keyword: the non-traditional security threats; prevention; countermeasures; Xinjiang

\begin{abstract}
It is an urgent task for researchers to study on the non-traditional security threats in Xinjiang. The author makes a summary of the status quo of study on the prevention and countermeasures against the non-traditional security threats in Xinjiang and finally produces a breakthrough in this study.

The domain and types of the non-traditional security threats in Xinjiang (henceforth, NSTX for short) are developing and changing all the time, which makes the non-traditional security issues in Xinjiang more complicated, diverse and uncertain. It is more and more difficult to make prevention against the non-traditional security threats. It is an urgent task for us to make research on the NSTX. It is known that only do we have a good knowledge of the previous studies and current situation we are likely to achieve success in the academic researches. In this sense, we should probe into the previous studies on the NSTX, analyze its status quo, and then we can make innovations in the study of this field and lay a solid foundation for the new theory.
\end{abstract}

\section{Generalization and Summarization: The Status Quo of the Study on the Prevention and Countermeasures against the Non-traditional Security Threats in Xinjiang}

First, some researchers have sorted out the situation of the non-traditional security in Xinjiang. Liu Yuezhen holds that Xinjiang is an important part of the national security strategy and makes analysis about energy security, environmental security, cultural security, terrorism which are the main challenges the non-traditional security in Xinjiang faces. In the perspective of cross-ethnic culture, Gao Rudong and Song Yizhi analyze the favorable conditions and unfavorable factors of the NSTX , and the great-leap-forward development of Xinjiang, the tradition of "harmony in diversity", the social consensus of the superior co-existence and the trends of the era all contribute to the handling of the NSTX and meanwhile, the "Three Evils Forces(terrorism, national secessionism and religious extremist)", the nationalism trends of neighboring countries, the fragile ecological environment and the economic backwardness make the situation in Xinjiang severe, or rather the prevention against the non-traditional security is relatively difficult. Xie Debo, Shang Chen and Zhu Hao tend to agree that the non-traditional security in Xinjiang includes the economic security, the "Three Evils", cultural security, environmental security and information security.

Second, some researchers have made analysis about the features of the non-traditional security in Xinjiang. Gao Yongjiu puts forward his opinion that the features of the non-traditional security threats around in the surrounding area of Xinjiang lie in several aspects, for instance, it is global, transnational, uncertain, non-military, transformative, and it needs cooperation from international community. An Xiaoping, Xie Guiping and Li Xinmin hold that the whole nation as well as Xinjiang is at high risk period full of outburst of social conflicts and development problems but the non-traditional security threat that Xinjiang needs to deal with is particular. The poverty, employment and crime issues are tagged with the ethnics, the ethnic culture rights and religion are magnified into the international community, the immigration, ecology and resource development issues become parochialism and national identity, ethnic identity and cultural identity become 
neterogeny issues.

Third, some researchers have put forward the countermeasures against the NSTX. Liu Yuezhen has found that the Scientific Outlook on Development is the solid foundation for us to work out the solutions to it. We need to enhance the economic foundation and the publicity on "Four Identities" so as to strength the national cohesion. Besides the crisis responding mechanism need to be set up. The political, law forces and armed forces should be improved. The cooperation between Central Asian countries and us should also be strengthened. While Shi Chunyan holds that we should face the challenges and the ethnic work should be paid much attention to and the people's livelihoods should be given priority in Xinjiang. We'd better strengthen the ideological work in Xinjiang, improve the ecological culture there, set up the non-traditional security threat responding mechanism and enhance the international cooperation and coordination. In Gao Rudong and Song Dezhi's point of view, it is sensible to accelerate the economic and social development in ethnic areas, spare no efforts in fighting against the "Three Evils Forces (terrorism, national secessionism and religious extremist)" and firmly repudiate the wrong ideas about the ethnic issues. What's more, cultivating the civic consciousness and cultural identity, and protection and improvement of the ecological environment in cross-border ethnic area are also a must.

Compared with the practice of the NSTX, the study on it is rare. Based on the resources collected by the author, no comprehensive academic monograph of the NSTX has been published in recent years. On the one hand, some studies which are pertaining to this field are not a comprehensive one, and they mainly went deep on issues like "Three Evils Forces (terrorism, national secessionism and religious extremist)", religion and ethnics. For instance, The Secession and Anti-secession in Xinjiang written by Chen Chao analyzes the historical factors of the emergence of secessionism in Xinjiang, looks back into the anti-secession fight in Xinjiang between the founding day of the PRC and the Cultural Revolutionary, and also the anti-secession fight since 1980s, probes into the anti-secession fight in the ideological field in Xinjiang and finally makes analysis about the trend of the anti-secession fight in Xinjiang and what to do with it. Li Qi focuses on one of the main factors that threaten the security of situation in Xinjiang, that is, Eastern Turkistan Secessionism and makes an overall analysis about it. The history, ideological system, features have been elaborated, and the practical impact and development tendency of it have been clear. In Li Qi's point of view, the ideological field is of great importance and fighting against Eastern Turkistan Secessionism is a part of international anti-terrorism efforts. Li Qi also makes researches on the attitude towards Eastern Turkistan Secessionism of some countries concerned and policies about it and puts forward suggestions on it. Zhong Minhe's book A True Xinjiang, looks back into the development and cultural integration in multi-ethnic area, the Xinjiang area in the provincial level since Qing Dynasty, the peaceful liberation of Xinjiang and its development, the establishment of ethnic autonomous region and the all-round development of economy and society in Xinjiang. Apart from these, Zhong criticizes the Eastern Turkistan Secessionism and affirms the efforts and outcome of anti-secessionism in China. As we know that "Three Evils Forces (terrorism, national secessionism and religious extremist)", religious and ethnic issues are the main factors that pose threat to the security in Xinjiang, and the whole nation as well as Xinjiang is at high risk period full of outburst of social conflicts and development problems, these issues and problems are mixed together which make the security situation in Xinjiang more complicated. The previous studies can be reference but their objective of study is relatively single one and it lacks in comprehensiveness and systematicness. Nowadays Xinjiang faces many non-traditional security threats, and the previous studies of this type make themselves limited and may cause difficulty if some policies concerned are going to be made. On the other hand, some studies and researches classify the NSTX as the category of Borderland Security and Borderland Government. Scholars like $\mathrm{Mu}$ Zhen and Ni Huifang, in The Social Stability and Development of Ethnic Area in Borderland, make researches on the a series of issues on the social stability and development in ethnic area based on survey and the analysis of the literature. The Study on China's Borderland Government written by Chen Lin gives new concept of borderland in the perspective of geography, history, ethnics, religion, identity, land power and sea power, development and national defense, and puts forward 7 issues, say the 
ethnic group in borderland, the religion in borderland, the identity of borderland, the land power in borderland, sea power in borderland, the development in borderland and defense in borderland. Chen also puts forward the governance mode, that is, "paying much attention to the inter-ethnic ideology while giving consideration to the regionalism, paying much attention to the ethnic ideology while giving consideration to the nationalism, paying much attention to gradient ideology while giving consideration to the geopolitical ideology, paying much attention to the micro-ideology while giving consideration to the marco-ideology and paying much attention to ruling ideology and managerialism. Zhu Qin probes into the governance issue in the borderland from the perspective of emergency management and puts forward the new mode, that is, harmonious governance, analyzes the foundation of governance, regulates the governance mode, determines the governance subject and key factors and makes suggestions on the emergency management training. These have certain reference significance for the prevention and countermeasures against the NSTX. Yu Xiaofeng, Xu Lili and Li Zhengyuan have written a book, The Introduction to Safety Science in Borderland,in this book, they make an overall elaboration focusing on the security issue in China's borderland in 3 chapters, and it sets a new milestone that cannot be ignored in China's borderland studies, especially in the borderland security issues. What's more, in this book the authors regard the non-traditional security issues in south Xinjiang as case study, probe into the emergence and significance of the study on the non-traditional security issues in south Xinjiang, sort out the issues of the non-traditional security in south Xinjiang and the countermeasures concerned, analyze the features and causes for the non-traditional security issues in south Xinjiang, and put forward the solutions to the improvement of the non-traditional security in south Xinjiang and suggestions on it. Just as Wang Yizhou has pointed out, "China has a vast territory, and situation in each region varies from each other. The non-traditional security issues vary from region to region in China, therefore, when determining the solutions, we should take the local situation into account together with the whole situation”. So does the prevention and countermeasures against the NSTX. The handling of the non-traditional security issues in China's borderland cannot fully reflect the particularity of the NSTX and even the non-traditional security threats in some areas of Xinjiang still cannot fully reflect the whole picture of the NSTX, which is a sort of deficiency of the study on the NSTX.

Generally speaking, there are four features of the study on the NSTX.

First, quite a lot studies focus on the concepts instead of practice. Nowadays, the non-traditional security issues cover a wide range of security issues. Sometimes the existing non-traditional security issue hasn't been solved yet, and a new one is emerging. And this situation usually leads to a superficially study on the unsolved existing non-traditional security issue, and of course a sensible and feasible solution is hard to be found. In recent years, researchers of non-traditional security issues have gradually realized this and shift their focus from the theoretical study, macroscopic study to the study on countermeasures and study on security issue in some specific fields. Although this trend is encouraging, the NSTX is diverse, the handling capability of the NSTX is inadequate, and Xinjiang is fragile to face a non-traditional security threat, in this sense, we still need to speed up the research on working out the specific, operational and practical solutions to the NSTX. There is still a big gap between the research progress and the reality.

Second, quite a lot studies focus on universality instead of particularity of Xinjiang. Based on the search results of Chinese academic literature online publishing database, there are 1992 pieces of academic articles focusing on non-traditional security while narrowing down the scope to non-traditional security in Xinjiang it has decreased from 1992 to 56. The national macroscopic studies are much more than the studies on Xinjiang, which results in retarding the improvement of the prevention and countermeasures against the NSTX. And nowadays the security incident happens more frequently than it was before and the consequence the security incident brings about become more and more severe.

Third, quite a lot studies focus on restoration instead of early warning mechanism, which is one of the problems of the study on the prevention and countermeasures against the NSTX. At the same time, the inadequate researches on forecast, prevention, pre-control and plan is the major problem 
of improvement of the prevention and countermeasures against the NSTX. The inadequacy lies in both the theory and practice, and this prevents us going deeper in the study on the NSTX, which reveals a lack of crisis awareness and unhealthy views on political achievement. And it also shows that people are not active in researching the forecast, prevention, pre-control and plan of the non-traditional security threats. Although this problem has been alleviated recent years, the restoration study still falls behind.

Fourth, quite a lot studies focus on single factor instead of multiple factors. A large number of studies focus on the single factor and go deeper into the non-traditional security threats that Xinjiang has to deal with from different aspects. This kind of studies probe into a certain non-traditional security threat, especially when a new threat emerges, these clear-cut studies can easily be applied into practice, but this kind of studies usually has two shortcomings. First of it, there will be a long list of the non-traditional security issues, which broadens the scope of it. Second, this kind of studies may ignore the fact that the NSTX are complex and it usually results from several factors and these factors interact with each other. If we make research of the non-traditional security issues based on a single factor, the research probably lacks the systematicness. Now when Xinjiang faces the many non-traditional security threats, only probing into one factor will not help us to figure out the whole picture of the NSTX, nor can we tackle the principal contradiction, nor can we lump factors concerned, nor can we solve the problems under different categories.

\section{Abstractness \& Extension: The Breakthrough of the Study on the Prevention and Countermeasures against the Non-traditional Security Threats in Xinjiang}

After several years' improvement, people's recognition of the NSTX has undergone a dramatic change. Its status, scale and connotation have changed a lot and formed its own characteristics. At the same time the increasing frequency of the non-traditional security incidents in Xinjiang create a contrast with the insufficient capability of preventing these from happening and dealing with the non-traditional security threats and its diversity, and this has become the major factor that hampers the stability and development in Xinjiang. The new era, special geographical conditions, complex cultural environment, and the complicated historical contradictions bring about many new features to the prevention and countermeasures against the NSTX. These new features and changes make the prevention and study become a more urgent task. In this sense, we should set up new concepts, adapt to the new situation and probe into the newly-emerging issues and finally we can hopefully make the breakthrough of the study on the prevention and countermeasures against the NSTX.

First, we should attach great importance to the study on the prevention and countermeasures against the NSTX. The practice of the prevention and countermeasures against the NSTX dates back to years ago, but the in-depth theoretical studies only began a little more than a decade ago. Now, the uneven development between the theoretical study and practice lies in the prevention and countermeasures against the NSTX. As early as years ago, there was the practice of the prevention and countermeasures against the NSTX, but the theoretical study on it falls behind. Till now many theoretical problems concerning the prevention and countermeasures against the NSTX remain unsolved. Much attention should be paid to this issue either theoretically or practically. There is a big gap between the study carried out by the developed provinces and its current situation and also a sharp contrast between the rich experience about the prevention and countermeasures against the NSTX and its current situation. The development and innovation of the theory has deep impact on the practice. The same is true of the prevention and countermeasures against the NSTX. In this sense, we need to strengthen the theoretical study. On the one hand, we should attach great importance to the study on the basic theory of the prevention and countermeasures against the NSTX , uncover the particularity of the NSTX , and work out the evolution mechanism of it. On the other hand, we should not neglect the significance of the study on the applied theory of the prevention and countermeasures against the NSTX. We need to carry out systematic study on the prevention, responding framework and policy by probing into its policy system, administration system, power system and strategy system. What's more, we should spare no efforts in working out the goal design, basic conception and capability composition of the prevention and countermeasures 
against the NSTX.

Second, we should attach great importance to the innovation of the countermeasures against the non-traditional security threat in Xinjiang. As we have already mentioned that quite a lot studies focus on the concepts instead of practice. If we want to solve this problem, the most effective way is to pay attention to the innovation of the countermeasures against the non-traditional security threat in Xinjiang. Being in the period of social transition, Xinjiang now faces unprecedented non-traditional security threats, the non-traditional security incidents happen now and then and many factors concerning potential non-traditional security threats gradually become active. The improvement of the prevention and countermeasures against the NSTX has entered into a critical period. Under such circumstance, much attention should be devoted to the study on the countermeasures against the NSTX. We need to integrate the non-traditional security theory with the particularity of Xinjiang and try to find out the feasible way to deal with this problem. If we work out the solution without taking the particularity of Xinjiang into account, probably this so-called solution will result in failure, which is the last thing we want to see.

At last, we should attach great importance to the research method of the prevention and countermeasures against the NSTX. Since the non-traditional security theory has been introduced to China, we have made some strides in the theoretical study and practice of the prevention and countermeasures against the NSTX. However, while we put the theory into practice, we have found that the traditional research perspective needs to be improved. Some theories cannot help us solve the problems of the prevention of threats and making countermeasures. Therefore, while we make full use of the traditional research method, keeping pace with times, discover the problems, analyzes the problems and solve the problems from a new perspective. The science and technology is constantly developing, and the traditional research method alone is not enough for us to cope with the ever-changing problem, even the traditional research method hampers the development of the study on the prevention and countermeasures against the NSTX, so the new method and new technology should be adopted to solve the problems that the traditional way fails in finding out the solution. By doing so, we are likely to make a great breakthrough and promote the development of the prevention and countermeasures against the NSTX and provide a more effective means to put the feasible theories into practice.

\section{Reference}

[1]Yuezhen Liu. Study on Challenges and Countermeasures of the Non-traditional Security Threats in Xinjiang. Economy and Culture of the Frontier, 2009(8)

[2]Rudong Gao, Yizhi Song. The Favorable Conditions and Unfavorable Factors in Xinjiang in Dealing with the Non-traditional Security Threats in the Perspective of Trans-boundary Nationalities. Journal of Tarim University,2011(3)

[3]Debo Xie, Chen Shang,Hao Zhu. A Study on Culture-Saveguard Borderland of Xinjiang Production and Construction Corps in the Perspective of Non-traditional Security Threats in Xinjiang.Journal of Party School of Xinjiang Production and Construction Corps,2010(4)

[4]Yongjiu Gao. The Non-traditional Security Issues of Xinjiang's Surrounding Area and Its Features.Xinjiang Social Science,2005(3)

[5]Xiaoping An,Guiping Xie, Xinming,Li. On Specific Characteristics of Non-traditional Security Threats in Xinjiang, Journal of Tarim University,2010(9)

[6]Chunyan Shi. The Focus of the Ethnic Work of Non-traditional Security in Xinjiang in New Century. Journal of Changchun University, 2012(1)

[7]Chao Chen. The Secession and Anti-secession in Xinjiang. Ethnics Press, Beijing,2009

[8]Qi Li. A Study on the East Turkistan Secessionism Power. China Social Science Press,Beijing, 2004 
[9]Minhe Zhong. A True Xinjiang. People Press,Beijing,2009

[10]Zhen Mu,.Huifang Ni. The Social Stability and Development of Ethnic Area in Borderland. China Social Science Press, Beijing, 1997

[11]Lin Chen. The Study on China’s Borderland Government. People Press,Kunming,2011

12]Qin Zhu. Harmonious Administration of Borderland Inhabited by the Minorities Nationalities-A Survey in the Perspective of Emergency Management. Yunnan People Press,Kunming,2010

[13]Xiaofeng Yu,Lili Xu,Zhengyuan,Li. The Introduction to Safety Science in Borderland, China Social Science Press,Beijing, 2013

[14]Yizhou Wang. China and Non-traditional Security. International Economics Review,2004(6) 